\title{
Medicina personalizada para el asma y la enfermedad pulmonar obstructiva crónica
}

\author{
Liam G. Heaney Lorcan P.A. McGarvey \\ Centre for Experimental Medicine, School of Medicine, Dentistry and Biomedical Sciences, Queens University Belfast, Belfast, Reino Unido
}

\author{
Palabras clave \\ Medicina personalizada · Medicina de precisión · Asma . \\ Enfermedad pulmonar obstructiva crónica · Comorbilidad
}

\section{Resumen}

El asma y la enfermedad pulmonar obstructiva crónica (EPOC) son condiciones prevalentes, y a pesar de los recientes avances y las múltiples terapias e intervenciones disponibles, persiste una necesidad clínica no satisfecha. En años recientes ha quedado claro que hay tanto una heterogeneidad significativa en cada uno de estos padecimientos como un traslape importante en muchas de sus características clínicas e inflamatorias. En paralelo, han emergido biomarcadores clínicos e inmunológicos útiles que alertan sobre el

pronóstico y la respuesta a la terapia para el asma y la EPOC. Estos biomarcadores nos permitirán tanto dirigir mejor los tratamientos existentes como identificar a los pacientes que responderán a las nuevas terapias disponibles. Los biomarcadores también facilitarán la identificación de nuevos blancos terapéuticos para desarrollos futuros. El uso de medicina de precisión en enfermedades de las vías respiratorias es ahora factible, y es un componente del cuidado a la salud personalizado en el asma y la EPOC.

(C) 2017 S. Karger AG, Basilea

\section{Introducción}

El asma es un síndrome caracterizado por síntomas variables, que incluyen sibilancias, disnea, opresión torácica y tos, en asociación con hiperreactividad bronquial y obstrucción reversible de las vías respiratorias [1]. La enfermedad pulmonar obstructiva crónica (EPOC) se caracteriza similarmente por síntomas que incluyen sibilancias, disnea, opresión torácica y tos, que usualmente es productiva y se asocia con una limitación fija del flujo de aire, una característica que ahora se incluye en la mayoría de las definiciones de la enfermedad [2]. Además de los síntomas crónicos, tanto el asma como la EPOC se asocian con una tendencia a agravarse de manera abrupta o subaguda. Estas «exacerbaciones» $\mathrm{o}$ «ataques» son eventos serios, y pueden ser fatales o por lo menos tener un gran impacto en la calidad de vida del paciente y consecuencias económicas considerables en la salud mundial $[3,4]$.
Persiste una necesidad no satisfecha en el asma y en la EPOC. Hasta $20 \%$ de los pacientes con asma no alcanzan un nivel de control aceptable, a pesar de recibir altas dosis de medicamentos antiinflamatorios y broncodilatadores, y su enfermedad se designa como «asma de difícil tratamiento» o «asma difícil» $[1,5]$. En la EPOC, aun en periodos entre exacerbaciones cuando los pacientes se consideran relativamente estables, los síntomas son prevalentes y problemáticos. En un estudio paneuropeo sobre pacientes con EPOC grave $\left(\mathrm{VEF}_{1}<50 \%\right.$ del valor predicho), más de $90 \%$ de los sujetos reportaron síntomas en algún grado todos los días [6]. Aun cuando la disnea fue el síntoma más frecuente, la tos y el esputo fueron particularmente problemáticos [7]. Cada vez se reconoce más la importancia de la tos en el estado de salud de los pacientes con EPOC [8], y hay gran necesidad de tratamientos dirigidos a controlar este síntoma [9].

El desarrollo de instrumentos de resultados reportados por los pacientes, tales como la Prueba de Evaluación de EPOC [10] y las information@karger.com

(c) 2020 S.Karger GmbH, Freiburg

www.karger.com/kxn

Karger ${ }^{\prime \prime}=$
Prof. Liam G. Heaney

Centre for Experimental Medicine

Wellcome-Wolfson Institute for Experimental Medicine

97 Lisburn Road, Belfast BT9 7BL, Reino Unido

1.heaney@qub.ac.uk 
herramientas EXACT (EXAcerbations of COPD Tool) [11] de resultados reportados por los pacientes, ha ayudado a capturar la gama de síntomas y problemas de salud que el tratamiento debe paliar. Adicionalmente, la recomendación actual de la iniciativa GOLD (Global Initiative for Chronic Obstructive Disease) de no categorizar a los pacientes sólo con base en la gravedad de la función pulmonar, sino en el riesgo de exacerbación y en la sintomatología, nos ayuda a superar una visión unidimensional y obsoleta de la EPOC como una enfermedad vinculada exclusivamente con la limitación fija del flujo de aire, para adoptar el concepto de un padecimiento con distintos fenotipos, que requieren opciones terapéuticas dirigidas de manera más precisa [2].

\section{Entender el problema clínico: un asunto personal}

Entender el problema clínico es un componente central del manejo personalizado de cualquier padecimiento. En pacientes con asma de difícil control, una evaluación sistemática y detallada a menudo identifica un problema coexistente $[12,13]$, ya sea solo (diagnóstico erróneo) o junto con asma leve/moderada, y cuando este problema se atiende efectivamente pueden controlarse los síntomas. También ha quedado claro que la comorbilidad es un componente importante en pacientes con EPOC [2, 14], y optimizar el manejo de cualquier comorbilidad, cuando sea posible, es esencial para obtener un nivel adecuado de control de la enfermedad. Es poco probable que la mala condición física y las enfermedades concomitantes respondan a tratamientos adicionales para la limitación del flujo de aire, y la evaluación clínica debe ser multidimensional, y es necesario reconocer el papel de factores extrapulmonares en el problema clínico global.

Un concepto atractivo propuesto hace poco es el de considerar la enfermedad de las vías respiratorias en términos de los llamados «rasgos tratables», que pueden identificarse y potencialmente atenderse $[15,16]$. Esto implica atender factores extrapulmonares que pueden mejorar la situación clínica, y además divide la enfermedad de las vías respiratorias en elementos que pueden evaluarse y abordarse individualmente. Asimismo, esto deconstruye la enfermedad de las vías respiratorias en partes componentes, incluyendo la inflamación de las vías respiratorias, hipersensibilidad al reflejo de la tos, daño estructural y lesiones en las vías respiratorias e hiperreactividad bronquial. Parece razonable, por tanto, que evaluar y abordar estos componentes en un nivel individual puede indicar al médico cómo pueden usarse los tratamientos de una manera más personal. Actualmente, algunos de estos rasgos son más «tratables» que otros; por ejemplo, la inflamación eosinofílica de las vías respiratorias es fácilmente modificable, mientras que, tradicionalmente, la hipersensibilidad al reflejo de la tos ha tenido una gama más limitada de opciones terapéuticas, aunque esto está cambiando [17]. El otro beneficio probable de este tipo de estrategia es que se evita el escalamiento progresivo del tratamiento más allá de cualquier expectativa razonable de beneficio terapéutico, lo cual es particularmente relevante en el uso de corticosteroides.

Un atributo inherente de este tipo de estrategia es el cuestionar las etiquetas dicotómicas tradicionales, tales como asma y EPOC, y el uso de un enfoque terapéutico único, fácilmente identificable en los lineamientos terapéuticos para el asma y la EPOC. En pacientes con asma sintomática persistente, con frecuencia hay un escalamiento progresivo en el tratamiento, particularmente en la terapia con corticosteroides, en un intento por controlar el asma. De hecho, la definición actual de asma grave requiere el escalamiento a un tratamiento con altas dosis (corticosteroides inhalados [CSI] en altas dosis más un segundo controlador en el año previo, o corticosteroides sistémicos $\geq 50 \%$ en el año previo), el cual podría mantener el asma bajo control o fracasar en ello [18]. El asma no controlada se define como síntomas persistentes de asma, exacerbaciones graves frecuentes que requieren al menos 2 ráfagas de corticosteroides sistémicos (u hospitalización) en el año previo o limitación persistente del flujo de aire [18]. El asma controlada que empeora durante la reducción progresiva del tratamiento con esteroides en dosis elevadas o durante el uso de terapia biológica adicional también cae en la categoría de enfermedad grave. Es importante recalcar que el diagnóstico de asma grave y refractaria sólo se aplica luego de una detallada evaluación multidisciplinaria para pacientes con asma en quienes se han excluido diagnósticos alternativos, se ha verificado la adherencia al tratamiento, se han tratado las comorbilidades y se han eliminado los factores desencadenantes (cuando es posible). Con la llegada de múltiples opciones terapéuticas nuevas para el asma grave en los próximos años (como se discute más adelante), lograr el diagnóstico correcto e identificar estos problemas centrales es más crítico que nunca, para evitar el uso inapropiado de estas terapias nuevas y potencialmente costosas.

De manera similar, aun cuando los CSI (en combinación con broncodilatadores de acción prolongada) han constituido la piedra angular de la terapia de mantenimiento para la EPOC moderada o grave por casi 20 años, su uso extendido, particularmente en dosis altas, se ha asociado indudablemente con eventos adversos [19]. A pesar de la abrumadora evidencia de que los esteroides inhalados reducen las exacerbaciones en la EPOC, la mayor preocupación es el aumento del riesgo de neumonía no fatal en algunos pacientes [20]. Evidencias recientes sugieren que puede alcanzarse un estado saludable, controlar los síntomas y prevenir exacerbaciones con una terapia sin esteroides, aun en casos de enfermedad moderada o grave [21]. Es evidente que no todos los pacientes con EPOC requieren tratamiento con CSI, y puesto que incluso puede ser nocivo para algunos, identificar qué pacientes deben recibir CSI es un problema crítico. Esto se discute con mayor detalle más adelante.

Hemos sabido por más de 50 años que los pacientes con asma y eosinofilia en el esputo responden al tratamiento con esteroides, como lo demostró elegantemente el Dr. Harry Morrow Brown luego de un ensayo clínico negativo de corticosteroides en pacientes con asma sin restricciones [22]. A pesar de esta incursión inicial en la medicina personalizada, nos ha tomado muchos años considerar el ya no usar dosis excesivas de esteroides en pacientes con enfermedad de las vías respiratorias.

Además del uso excesivo del tratamiento con esteroides inhalados en la enfermedad de las vías respiratorias, existe la cuestión de la 
Tabla 1. Comorbilidades comunes en asma y EPOC graves

\begin{tabular}{ll}
\hline Asma & EPOC \\
\hline $\begin{array}{ll}\text { Comorbilidades pulmonares: apnea del sueño obstructiva, síndrome de } \\
\text { hiperventilación, tabaquismo/enfermedad relacionada con el tabaquismo }\end{array}$ & $\begin{array}{l}\text { Comorbilidades pulmonares: apnea del sueño obstructiva, } \\
\text { bronquiectasia, fibrosis intersticial }\end{array}$ \\
\hline Obesidad y mala condición física & Obesidad y mala condición física \\
\hline Comorbilidades gastrointestinales: enfermedad de reflujo gastroesofágico & $\begin{array}{l}\text { Comorbilidades gastrointestinales: reflujo gastroesofágico, } \\
\text { enfermedad de úlcera péptica }\end{array}$ \\
\hline $\begin{array}{l}\text { Factores sicológicos: rasgos de la personalidad, percepción de los síntomas, } \\
\text { ansiedad, depresión }\end{array}$ & $\begin{array}{l}\text { Factores sicológicos: percepción de los síntomas y manejo } \\
\text { de la apnea, ansiedad, depresión }\end{array}$ \\
$\begin{array}{l}\text { Vías respiratorias superiores: rinosinusitis/pólipos nasales (adultos), } \\
\text { disfunción de las cuerdas vocales }\end{array}$ & $\begin{array}{l}\text { Factores cardiovasculares: arteriopatía coronaria, insuficiencia cardiaca } \\
\text { congestiva, fibrilación auricular, hipertensión }\end{array}$ \\
\hline $\begin{array}{l}\text { Intolerancia a fármacos incluyendo la aspirina, antiinflamatorios } \\
\text { no esteroides, bloqueadores } \beta \text {-adrenérgicos, inhibidores } \\
\text { de la enzima convertidora de angiotensina }\end{array}$ & $\begin{array}{l}\text { Factores metabólicos/sistémicos: hiperglucemia, bajo peso corporal, } \\
\text { obesidad, osteoporosis }\end{array}$ \\
\hline $\begin{array}{l}\text { Influencias hormonales: periodo premenstrual, menarquia, menopausia, } \\
\text { trastornos tiroideos }\end{array}$ & $\begin{array}{l}\text { Factores oncológicos: cáncer de pulmón, cáncer esofágico, cáncer } \\
\text { pancreático }\end{array}$ \\
\hline
\end{tabular}

falta de adherencia a la terapia de mantenimiento en pacientes que responderían bien a ella. Éste es un ejemplo adicional de cómo una estrategia personalizada es crítica al atender el problema clínico. En un nivel simple, la falta de adherencia puede ser no intencional, cuando los pacientes quieren tomar el tratamiento pero se ven impedidos por barreras fuera de su control, como la mala memoria para recordar instrucciones, la falta de comprensión, la dispraxia o mala coordinación, olvidos o carencia de recursos económicos. Esto es muy diferente de la falta de adherencia intencional, cuando un paciente decide no tomar el tratamiento debido a creencias (a menudo falsas) sobre la medicación, a un estado de negación, a ganancias secundarias o creencias sobre la enfermedad. Una estrategia única está destinada a fracasar en estos pacientes, dada la complejidad y extensión de este problema clínico. La falta de adherencia es prevalente en pacientes que presentan asma difícil [23, 24], y no es sencillo identificarla en la práctica clínica rutinaria, lo que puede llevar a un mal resultado y a la muerte. Otro efecto es que algunos pacientes son diagnosticados erróneamente con enfermedad «refractaria», con la posibilidad de un escalamiento inapropiado a corticosteroides sistémicos o terapias biológicas.

Algunas evaluaciones «basadas en biomarcadores» de la respuesta a los corticosteroides en el asma grave desarrolladas recientemente podrían identificar pacientes que deberían alcanzar un mejor control del asma con una adherencia más fiel al tratamiento estándar [25]. El desarrollo de nuevas tecnologías de «inhaladores inteligentes» junto con estas evaluaciones basadas en marcadores permitirán identificar y atender en la clínica el problema de la falta de adherencia. En la EPOC, la adherencia también es subóptima. Como ocurre con el asma, es un fenómeno multifactorial, e incluye la incapacidad de recoger las prescripciones para el medicamento [26] y una mala técnica al usar el inhalador [27]. En un estudio reciente, Sulaiman et al. investigaron la naturaleza y la extensión de la falta de adherencia en pacientes dados de alta del hospital luego de una exacerbación de EPOC [28]. Empleando un dispositivo acústico integrado en un inhalador de salmeterol/ fluticasona, que cuantificó qué tan bien y con qué frecuencia los pacientes se administraban el medicamento, encontraron que solamente $7 \%$ alcanzaron una adherencia de más de $80 \%$. De manera poco sorpresiva, los pacientes más ancianos, con función pulmonar más afectada y con deterioro cognitivo tuvieron mayores probabilidades de mostrar mala adherencia y cometer errores frecuentes en la técnica al usar el inhalador.

Una cuestión clave tanto para el asma como para la EPOC es cómo manejar efectivamente la falta de adherencia en este grupo de pacientes [29], pero el punto de partida es identificar que la falta de adherencia es el problema clínico en un paciente que debería responder bien al tratamiento con esteroides inhalados. Asimismo, es probable que las nuevas tecnologías de vigilancia, con recordatorios para el usuario en «tiempo real» y retroalimentación sobre la técnica pronto estén disponibles y permitan aplicar nuevas estrategias personalizadas para este problema sustancial.

\section{Comorbilidad en asma y EPOC}

Varias comorbilidades se reportan comúnmente en poblaciones con asma y EPOC grave (Tabla 1), y los lineamientos de manejo recomiendan el manejo clínico de estas comorbilidades $[2,18]$. En la EPOC, algunas de éstas, como la arteriopatía coronaria, la insuficiencia cardiaca y el cáncer de pulmón, se asocian fuertemente con una alta mortalidad, mientras que otras, como la hipertensión, diabetes, apnea del sueño y reflujo, pueden hacer más complejo el tratamiento de la enfermedad en pacientes individuales [30].

Sin embargo, no hay mucha evidencia de que tratar comorbilidades como el reflujo gastroesofágico tenga un impacto mayor en el resultado clínico en estas enfermedades [31, 32]. Esto puede ser porque persiste el reflujo no-ácido o porque la presencia de reflujo gastroesofágico tiene poco efecto en la enfermedad de las vías respiratorias subyacente; sin embargo, comúnmente se le mencio-
12

Kompass Neumol 2020;2:10-17 DOI: $10.1159 / 000504517$ 
na como causa de tos crónica. El reflujo gastroesofágico puede tratarse quirúrgicamente de manera exitosa mediante funduplicatura, y se ha sugerido su eficacia en el asma [33], pero hasta que hagamos estudios de funduplicatura con controles de operación simulada puede ser difícil discernir la relación causal entre el reflujo y la enfermedad de las vías respiratorias. Este panorama muestra un contraste marcado con la evidencia a favor de la rehabilitación pulmonar en EPOC, que ha sido consistentemente positiva [34], confirmando que la intervención sobre la morbilidad extrapulmonar tiene valor en el manejo de la enfermedad de las vías respiratorias, y estos programas terapéuticos están evolucionando hacia un enfoque más centrado en el paciente. Un problema clave para el avance en el manejo de muchos de estos factores extrapulmonares será el dilucidar la asociación de «causa y efecto» y dirigir las intervenciones con base en la evidencia, para ofrecer un manejo verdaderamente personalizado.

\section{Heterogeneidad en la inflamación de las vías respiratorias}

Datos recientes en pacientes con asma leve o grave sugieren que hay evidencia de una heterogeneidad significativa en la enfermedad $[35,36,37,38,39]$, y un examen de las firmas genéticas en cohortes bien caracterizadas de pacientes con asma grave ha demostrado que entre 25 y $50 \%$ de los pacientes tienen una firma genética prototípica de citocinas tipo 2 (T2), la llamada «enfermedad con T2 alta» (con niveles elevados de IL-4, - 5 y -13 y eosinofilia en las vías respiratorias, a pesar de la adherencia al tratamiento con corticosteroides). En pacientes con asma grave sin evidencia de inflamación T2 («asma con T2 baja»), es probable que la dosis de corticosteroides se haya escalado inapropiadamente en un intento por manejar síntomas persistentes que no responden a los corticosteroides. Dada la evidencia de que la respuesta a los corticosteroides está confinada a la enfermedad con T2 alta [35, $40,41]$, una cuestión clave para el manejo del asma grave en el futuro es desarrollar pruebas objetivas y algoritmos de manejo validados no solamente para iniciar el tratamiento con corticosteroides, sino para permitir al médico determinar los casos en que dosis mayores de corticosteroides no producirán una respuesta clínica adicional. Abandonar el escalamiento en la terapia con corticosteroides que actualmente se recomienda con base en los síntomas será un componente fundamental del «tratamiento personalizado» para el asma grave en el futuro, y facilitará la optimización de la dosis de corticosteroides. También permitiría diagnosticar el asma grave sin escalar la terapia con corticosteroides más allá de un punto donde, en muchos casos, es poco probable que haya un beneficio terapéutico.

El mismo problema de riesgo contra beneficio es evidente con respecto al uso de CSI en la EPOC. Como se discutió anteriormente, los CSI se indican usualmente para reducir las exacerbaciones, y los primeros ensayos clínicos en EPOC no estratificaron a los pacientes con base en su fenotipo inflamatorio. En consecuencia, los CSI se prescriben ampliamente en casos de EPOC a pesar de que hay poca información que indique al médico quiénes realmente se beneficiarán de ellos sin efectos adversos. Evidencias recientes sugieren que el recuento sanguíneo de eosinófilos puede ayudar al médico a tomar esta decisión. En un metaanálisis agrupado de 10 ensayos llevados a cabo por GlaxoSmithKline en los que se disponía de datos basales de recuento sanguíneo de eosinófilos y de eventos de neumonía, Pavord et al. [42] calcularon el número de eventos de neumonía estratificados con respecto al recuento basal de eosinófilos en la sangre $(<2$ y $\geq 2 \%$ de los leucocitos sanguíneos). Encontraron que los pacientes con menores recuentos basales de eosinófilos (es decir, $<2 \%$ ) tenían mayor riesgo de desarrollar neumonía, y éste era independiente del uso de CSI. Por tanto, un recuento bajo de eosinófilos sanguíneos puede añadirse a la lista de factores de riesgo preexistentes conocidos para la neumonía (edad, obstrucción grave de las vías respiratorias, bronquiectasia coexistente y neumonía previa), y puede tomarse en consideración para decidir si debe iniciarse la terapia de esteroides inhalados o no. Sin embargo, iniciar un tratamiento es muy distinto de suspender un medicamento; debe evitarse cualquier precipitación para descontinuar los CSI sin una consideración cuidadosa. En un análisis post hoc publicado recientemente sobre el ensayo de Retiro de Esteroides Inhalados durante el Manejo Optimizado de Broncodilatador (Withdrawal of Inhaled Steroids during Optimized Bronchodilator Management, WISDOM), los pacientes con un recuento basal de eosinófilos sanguíneos más alto ( $\geq 4 \%$ o $\geq 300$ células por $\mu$ l) tuvieron una probabilidad significativamente mayor de sufrir exacerbaciones moderadas a graves luego de suspender los CSI [43]. El recuento de eosinófilos sanguíneos es un biomarcador relativamente fácil de obtener en la clínica, aunque aún se desconoce hasta qué punto distingue con exactitud a los pacientes con probabilidades de beneficiarse de aquellos en riesgo de sufrir daño. Sin embargo, se requieren trabajos adicionales para validar una estrategia de ajuste de esteroides con base en el recuento de eosinófilos sanguíneos en la EPOC, incluyendo un estudio prospectivo sobre el retiro de esteroides inhalados en sujetos con valores bajos del biomarcador, junto con una caracterización precisa del fenotipo inflamatorio de los eventos de exacerbación.

Casi con certeza, la estrategia anterior es un abordaje «básico» de la heterogeneidad inflamatoria en la enfermedad de las vías respiratorias, al caracterizar la inflamación de las vías respiratorias como eosinofílica o no-eosinofílica. Un problema clave que debe atenderse en el futuro es determinar si esta heterogeneidad fenotípica tanto en asma como en EPOC puede subdividirse adicionalmente en vías mecanicistas discretas o endotipos [44]. Es enteramente factible que varias vías biológicas diferentes puedan dar origen a la eosinofilia de las vías respiratorias, y ya se están abordando mecanismos específicos en el asma mediante el bloqueo de citocinas T2 específicas.

En el asma grave, estos nuevos fármacos se han administrado a pacientes con enfermedad con T2 alta, quienes padecen asma eosinofílica refractaria a pesar de recibir dosis altas de CSI. El omalizumab es un anticuerpo monoclonal humanizado derivado de DNA, de tipo inmunoglobulina (Ig) G1K, que se une selectivamente a la IgE humana en el mismo sitio que el receptor de IgE de alta afinidad, formando complejos inmunes con la IgE libre [45]. 
Esta unión inhibe la interacción de la IgE con los receptores de IgE en la superficie de mastocitos, basófilos y otros tipos celulares, impidiendo la liberación de mediadores inflamatorios que ocurre en el asma alérgica. Los ensayos clínicos han demostrado una reducción en el número de visitas de emergencia no programadas e ingresos al hospital, y los lineamientos actuales recomiendan el uso de omalizumab como terapia complementaria en el asma grave [45]. De manera interesante, dada la falta de eficacia y el abandono de algunas otras nuevas terapias anti-IgE (p. ej., quilizumab, MEDI-4212 [46]), la observación de que algunos pacientes alérgicos con asma grave no responden bien al omalizumab potencialmente permitirá explorar el mecanismo de acción y definir a un «respondedor» con base en vías inflamatorias específicas.

Recientemente llegaron a la clínica tratamientos dirigidos a la interleucina (IL)-5 (mepolizumab, reslizumab), y se ha demostrado que reducen a la mitad el número de exacerbaciones graves en sujetos con asma eosinofílica, con efectos más modestos en los síntomas y la función pulmonar $[47,48]$. También están bajo estudio terapias dirigidas al receptor de la IL-5 (IL-5R) (benralizumab), así como a la IL-13 (lebrikizumab, tralokinumab) y al IL-4Ra (dupilumab). El ensayo clínico de fase 3 del fármaco anti-IL-13 lebrikizumab ha demostrado una eficacia menor de la que se anticipaba en los estudios de fase $2 \mathrm{y}$, aunque es decepcionante en cierto nivel, esto sugiere que el papel de la IL-13 no es tan crucial en el asma más grave y casi con certeza nos brindará una mayor comprensión del papel de esta citocina en el asma [49]. Hay otras estrategias dirigidas al eje $\mathrm{T} 2$, incluyendo el antagonismo con la prostaglandina D2 [50], y parece probable que todas estas terapias se dirigirán a grupos de pacientes que se superponen parcialmente; identificar qué pacientes responden mejor a diferentes clases de fármacos podría requerir estudios comparativos, y entender los diferentes subgrupos respondedores será una tarea clave cuando estos fármacos estén disponibles en la clínica. Hay la intrigante posibilidad de que los estudios comparativos se acompañen con una evaluación mecanicista del efecto del fármaco; el empleo de biomarcadores predictivos de la respuesta clínica mejorará aún más nuestra comprensión de la variación genotípica en el asma.

Con respecto a la EPOC, dada la evidencia de que la inflamación eosinofílica desempeña un papel en algunos pacientes con la enfermedad, se están ensayando terapias dirigidas al eje de citocinas $\mathrm{T} 2$ en esta población, que generalmente se estratifica utilizando un marcador de la biología eosinofílica, como el recuento de eosinófilos en la sangre periférica. En un estudio reciente controlado por placebo del benralizumab (un anticuerpo monoclonal anti-IL$5 R \alpha$ ) en pacientes con EPOC moderada a grave y eosinofilia, no se encontró reducción en el número de exacerbaciones agudas [51]. Sin embargo, se observó una disminución en la frecuencia de exacerbaciones agudas y una pequeña mejoría en la función pulmonar y la calidad de vida en el subgrupo especificado con concentraciones basales de eosinófilos de 200 por $\mu$ l o más [51]. Parece probable que el grado de heterogeneidad en términos de biología inflamatoria es mayor en la EPOC, pero entender la naturaleza y la extensión del efecto clínico de la inhibición de citocinas T2 en la EPOC no sólo podría ofrecer un avance que sería bienvenido en la clínica, sino nos permitiría adelantar un poco en nuestra comprensión de la heterogeneidad inflamatoria en la EPOC y en otros componentes de la enfermedad que requieren atención.

Entre las estrategias antiinflamatorias alternativas en la EPOC se ha propuesto abordar el desequilibrio de proteasas en las vías respiratorias. Las metaloproteasas de matriz tienen efectos que dañan los tejidos en la enfermedad pulmonar y están implicadas en el desarrollo de la EPOC, en particular en el subfenotipo de enfisema. Claramente, es importante inhibir tales acciones tan pronto como sea posible, y la llegada de mejores técnicas de imagen puede dar origen a biomarcadores que ayuden a seleccionar a los pacientes idóneos para el tratamiento $[52,53]$.

\section{Vinculando la necesidad no satisfecha y el tratamiento personalizado en la enfermedad de las vías respiratorias}

A pesar del advenimiento de nuevos tratamientos anticitocinas T2, se reconoce que aún habrá necesidades no cubiertas en la enfermedad respiratoria. Un objetivo importante de la investigación sobre el asma es el manejo terapéutico de las facetas bajas en T2 del asma, que no responden a los corticosteroides, particularmente en la enfermedad grave. Ahora parece claro que los síntomas y la fisiología alterada en el asma se manifiestan en la ausencia de inflamación eosinofílica/citocinas T2, pero no es evidente cuáles son los mecanismos fisiopatológicos subyacentes a estos procesos. Las posibilidades incluyen un proceso inflamatorio diferente o problemas estructurales no-inflamatorios, como una contractibilidad anormal del músculo liso, señalización epitelial aberrante o infección de las vías respiratorias. Entender la extensión y el mecanismo de la enfermedad con T2 baja en el asma grave será un objetivo importante en la investigación de la próxima década.

El papel de la IL-17 en el asma ha generado interés, y un estudio inicial que utilizó un fármaco anti-IL-17 (brodalumab) sugirió alguna eficacia en pacientes asmáticos con reversibilidad broncodilatadora significativa [54], pero hace falta establecer el papel preciso de la IL-17 en el asma grave. Datos preliminares empleando terapia con macrólidos en pacientes con bajo recuento de eosinófilos periféricos también han sugerido una reducción en las exacerbaciones, pero aún se debe clarificar si se trata de un efecto antibacteriano o de tipo antiinflamatorio [55]. La termoplastia bronquial ya está disponible como intervención terapéutica en el asma [56], pero identificar qué pacientes individuales se beneficiarán con cualquiera de estas intervenciones es un problema clave. Sin embargo, optimizar el tratamiento con corticosteroides y la disponibilidad de nuevas terapias con blancos específicos brindará grandes oportunidades de ofrecer tratamiento personalizado para un paciente específico y para identificar nuevos mecanismos en la enfermedad grave. Se están explorando nuevas estrategias para optimizar la dosis de corticosteroides en el asma grave, utilizando biomarcadores combinados, y si son exitosas, será fácil transferirlas a la práctica clínica de rutina [57].

En la EPOC, un área clave de necesidad no cubierta es el tratamiento de pacientes con enfermedad grave que no es probable que responda a las terapias convencionales. En el enfisema más avan-
14

Kompass Neumol 2020;2:10-17 DOI: $10.1159 / 000504517$ 
zado, donde se presenta un daño estructural y funcional significativo, hay la necesidad de identificar a los pacientes con posibilidades de beneficiarse con una cirugía de reducción de volumen pulmonar (CRVP). En el Ensayo Nacional de Tratamiento del Enfisema (National Emphysema treatment Trial, NETT) original, quedó claro que no todos los pacientes inicialmente aleatorizados se beneficiaron con la CRVP [58]. Se identificaron subgrupos de respondedores (enfisema predominante del lóbulo superior, con poca capacidad para el ejercicio a pesar de la rehabilitación pulmonar), resaltando una vez más la naturaleza personalizada de la terapia para la EPOC. Estos hallazgos han ayudado a precisar el diseño de ensayos de reducción broncoscópica del volumen pulmonar (RBVP), que involucran la colocación de válvulas endobronquiales unidireccionales. Estudios recientes han mostrado que este tratamiento puede ser adecuado para pacientes con enfisema de distribución más homogénea que experimentaron malos resultados en el ensayo NETT [59].

El mayor desafío futuro en la enfermedad asmática de las vías respiratorias sigue siendo idear una terapia que modifique la enfermedad. En el asma, es tentador especular que si pudiéramos entender por qué los pacientes con un tipo particular de la enfermedad (con T2 alta/eosinofílica), que usualmente responde a dosis bajas de CSI, se vuelven «relativamente» resistentes a los corticosteroides y requieren altas dosis (con frecuencia sistémicas), podríamos abordarlos terapéuticamente. Esta área ha sido tema de estudio por muchos años, pero no ha emergido aún un mecanismo preciso, evidenciado por una demostración terapéutica. En un estudio aleatorizado y controlado de retiro del omalizumab (el estudio de Evaluación de la Persistencia de la Respuesta a Xolair después de Terapia de Largo Plazo, Evaluating Xolair Persistency of Response after Long-Term Therapy, XPORT), se administró aleatoriamente placebo o se continuó el omalizumab en 176 pacientes que habían recibido el fármaco por al menos 5 años [60]. El grupo del placebo tuvo un tiempo más corto antes de la primera exacerbación que quienes continuaron con el omalizumab; sin embargo, es notable que $47.7 \%$ de los pacientes con placebo no tuvieron exacerbación durante el periodo de seguimiento de un año, y es interesante especular que algunos pacientes tuvieron un beneficio persistente; no obstante, se requieren estudios y análisis adicionales para determinar si éste es el caso. Debe mencionarse también que más de $25 \%$ de los pacientes aleatorizados no recibían CSI al momento de la aleatorización, y no queda claro si estos datos pueden extrapolarse a pacientes con asma grave que toman omalizumab y requieren además tratamiento frecuente con dosis altas de corti- costeroides para mantener la enfermedad bajo control. Puede concluirse que, como grupo, los pacientes que suspenden el tratamiento con omalizumab luego de usarlo a largo plazo tienen resultados menos buenos que quienes continúan el tratamiento.

La modificación de la enfermedad en la EPOC es quizá una dificultad mayor que en el asma, por varias razones. Primero, se sabe considerablemente menos sobre la fisiopatología de la EPOC y, en consecuencia, el desarrollo y empleo de tratamientos dirigidos a vías inmunes específicas ha sido mucho más lento. Segundo, la EPOC tiende a presentarse en pacientes más viejos, a menudo cuando está muy avanzada y quizá no sea susceptible a intervenciones que modifiquen la enfermedad. Claramente, se necesitan más trabajos para entender los eventos en edades tempranas que contribuyen al desarrollo de la EPOC, mejorar nuestra capacidad para identificar la enfermedad temprana, y dentro de ese grupo, seleccionar y tratar a quienes tengan mayores probabilidades de sufrir un deterioro rápido de la función pulmonar. Finalmente, evitar los factores ambientales (notablemente, el tabaquismo y la quema de combustibles de biomasa) que son causas obvias de la EPOC es un desafío enorme $[61,62]$. Un área de interés emergente es el potencial de regeneración pulmonar mediante el uso de células madre mesenquimales. A pesar del prometedor trabajo preclínico en este campo, sólo se ha emprendido un ensayo clínico controlado con placebo de células madre mesenquimales administradas sistemáticamente a pacientes con EPOC moderada a grave [63]. Aunque el tratamiento aparentemente fue seguro, no se observó efecto antiinflamatorio ni mejoró la función pulmonar, lo que sugiere que la aplicación clínica de esta modalidad de tratamiento particular para pacientes con enfermedad más grave aún es lejana.

\section{Conclusión}

La enfermedad de las vías respiratorias es heterogénea, y un enfoque uniforme para su diagnóstico y manejo, aunque brinda beneficios en algunos niveles, también ha llevado a resultados discordantes en subgrupos de pacientes debido a la heterogeneidad de la enfermedad. Una evaluación clínica precisa, «personalizada», con énfasis particular en comprender el problema clínico, atender la morbilidad extrapulmonar y manejando las dificultades conductuales, como la falta de adherencia al tratamiento con esteroides inhalados, es tan crítica para la medicina de precisión en las enfermedades de las vías respiratorias como elegir el fármaco correcto para el paciente correcto.

\section{Referencias}

1 Global Initiative for Asthma: Global strategy for asthma management and prevention. http://ginasthma.org/2016-gina-report-globalstrategy-for-asthma-management-and-prevention/ (accessed December 20, 2016).

2 Global Initiative for Chronic Obstructive Lung Disease (GOLD): Global strategy for the diagnosis, management and prevention of COPD. 2016. http://goldcopd.org/.
Serra-Batlles J, Plaza V, Morejón E,et al.: Costs of asthma according to the degree of severity. Eur Respir J 1998;12:1322-1326.

4 Foo J, Landis SH, Maskell J, et al.: Continuing to confront COPD international patient survey: economic impact of COPD in 12 countries. PLoS One 2016;11:e0152618.
Hekking PP, Wener RR, Amelink M, et al.: The prevalence of severe refractory asthma. J Allergy Clin Immunol 2015;135:896-902.

6 Kessler R, Partridge MR, Miravitlles M, et al.: Symptom variability in patients with severe COPD: a pan-European cross-sectional study. Eur Respir J 2011;37:264-272. 
7 Burgel PR, Wedzicha JA: Chronic cough in chronic obstructive pulmonary disease: time for listening? Am J Respir Crit Care Med 2013 187:902-904.

$>8$ Polley L, Yaman N, Heaney L, et al.: Impact of cough across different chronic respiratory diseases: comparison of two cough-specific health-related quality of life questionnaires. Chest 2008;134:295-302.

$\checkmark 9$ Calverley PM: Cough in chronic obstructive pulmonary disease: is it important and what are the effects of treatment? Cough 2013;9:17.

10 Jones PW, Harding G, Berry P, et al.: Development and first validation of the COPD Assessment Test. Eur Respir J 2009;34:648-654.

11 Leidy NK, Murray LT, Monz BU, et al.: Measuring respiratory symptoms of COPD: perfor mance of the EXACT-Respiratory Symptoms Tool (E-RS) in three clinical trials. Respir Res 2014;15:124.

12 Heaney LG, Robinson DS: Severe asthma treatment: need for characterising patients. Lancet 2005;365:974-976.

13 Heaney LG, Conway E, Kelly C, Jet al.: Predictors of therapy resistant asthma: outcome of a systematic evaluation protocol. Thorax 2003; 58:561-566.

14 Cleutjens F, Triest F, Wilke S, Vet al.: New insights in chronic obstructive pulmonary disease and comorbidity. Am J Respir Crit Care Med 2015;191:1081.

15 Agusti A, Bel E, Thomas M, et al.: Treatable traits: toward precision medicine of chronic airway diseases. Eur Respir J 2016;47:410-419.

16 Gonem S, Raj V, Wardlaw AJ, et al.: Phenotyping airways disease: an A to $\mathrm{E}$ approach. Clin Exp Allergy 2012;42:1664-1683.

17 Abdulqawi R, Dockry R, Holt K, et al.: P2X3 receptor antagonist (AF-219) in refractory chronic cough: a randomised, double-blind, placebo-controlled phase 2 study. Lancet 2015; 385:1198-1205.

-18 Chung KF, Wenzel SE, Brozek JL, et al.: International ERS/ATS guidelines on definition, evaluation and treatment of severe asthma. Eur Respir J 2014;43:343-373.

- 19 Flynn RW, MacDonald TM, Hapca A,et al.: Quantifying the real life risk profile of inhaled corticosteroids in COPD by record linkage analysis. Respir Res 2014;15:141.

20 Calverley PM, Anderson JA, Celli B, et al.: Salmeterol and fluticasone propionate and survival in chronic obstructive pulmonary disease. N Engl J Med 2007;356:775-789.

-21 Wedzicha JA, Banerji D, Chapman KR, Vet al.; FLAME Investigators: Indacaterol-glycopyrronium versus salmeterol-fluticasone for COPD. N Engl J Med 2016;374:2222-2234.

22 Brown HM: Treatment of chronic asthma with prednisolone: significance of eosinophils in the sputum. Lancet 1958;2:1245-1247.

23 Gamble J, Stevenson M, McClean E, Heaney LG: The prevalence of non-adherence in difficult asthma. Am J Respir Crit Care Med 2009; 180:817-822.

24 Murphy AC, Proeschal A, Brightling CE, et al.: The relationship between clinical outcomes and medication adherence in difficult-to-control asthma. Thorax 2012;67:751-753.
25 McNicholl DM, Stevenson M, McGarvey LP, Heaney LG: The utility of fractional exhaled nitric oxide suppression in the identification of nonadherence in difficult asthma. Am J Respir Crit Care Med 2012;186:1102-1108.

26 Blackstock FC, ZuWallack R, Nici L, Lareau SC: Why don't our patients with chronic obstructive pulmonary disease listen to us? The enigma of nonadherence. Ann Am Thorac Soc 2016;13:317-323.

27 Press VG, Arora VM, Shah LM, et al.: Misuse of respiratory inhalers in hospitalized patients with asthma or COPD. J Gen Intern Med 2011; 26:635-642.

28 Sulaiman I, Cushen B, Greene G, et al.: Objective assessment of adherence to inhalers by COPD patients. Am J Respir Crit Care Med 2016, Epub ahead of print.

29 Nieuwlaat R, Wilczynski N, Navarro T, et al.: Interventions for enhancing medication adherence. Cochrane Database Syst Rev 2014;11: $1-730$.

30 Divo M, Cote C, de Torres JP, et al.; BODE Collaborative Group: Comorbidities and risk of mortality in patients with chronic obstructive pulmonary disease. Am J Respir Crit Care Med 2012;186:155-161.

31 Gibson PG, Henry RL, Coughlan JL: Gastrooesophageal reflux treatment for asthma in adults and children. Cochrane Database Syst Rev 2003;2:1-33.

32 Ingebrigtsen TS, Marott JL, Vestbo J, Net al.: Gastro-esophageal reflux disease and exacerbations in chronic obstructive pulmonary disease. Respirology 2015;20:101-107.

33 Rothenberg SS, Bratton D: The effects of laparoscopic Nissen fundoplication to enhance pulmonary function in the treatment of a patient with severe asthma and gastroesophageal reflux disease. J Allergy Clin Immunol 2008; 121:1069-1070.

34 Lacasse Y, Martin S, Lasserson TJ, Goldstein RS: Meta-analysis of respiratory rehabilitation in chronic obstructive pulmonary disease. A Cochrane systematic review. Eura Medicophys 2007;43:475-485.

35 Woodruff PG, Modreck B, Choy DF, et al.: Thelper type 2-driven inflammation defines major subphenotypes of asthma. Am J Respir Crit Care Med 2009; 180:388-395.

36 Jia G, Erickson RW, Choy DF, et al.: Periostin is a systemic bio-marker of eosinophilic airway inflammation in asthmatic patients. J Allergy Clin Immunol 2012;130:647-654.

37 Butler CA, McQuaid S, Taggart CC, et al.: Glucocorticoid receptor $\beta$ and histone deacetylase 1 and 2 expression in the airways of severe asthma. Thorax 2012;67:392-398

38 Shikotra A, Choy DF, Ohri CM, et al.: In creased expression of immunoreactive thymic stromal lymphopoietin in patients with severe asthma. J Allergy Clin Immunol 2012;129: 104-111.

39 Choy D, Shikotra A, Siddiqui S, et al.: Mutually exclusive Th2 and Th17 bronchial gene expression signatures are associated with eosinophilia in asthma. Eur Respir J 2014;44(suppl 58):P3868.

40 McGrath KW, Icitovic N, Boushey HA, et al.: A large subgroup of mild-to-moderate asthma is persistently non-eosinophilic. Am J Respir Crit Care Med 2012;185:612-619.
1 Pavord ID, Brightling CE, Woltmann G, Wardlaw AJ: Non-eosinophilic corticosteroid unresponsive asthma. Lancet 1999;353;2213-2214.

42 Pavord ID, Lettis S, Anzueto A, Barnes N Blood eosinophil count and pneumonia risk in patients with chronic obstructive pulmonary disease: a patient-level meta-analysis. Lancet Respir Med 2016;4:731-741.

43 Watz H, Tetzlaff K, Wouters EF, et al.: Blood eosinophil count and exacerbations in severe chronic obstructive pulmonary disease after withdrawal of inhaled corticosteroids: a posthoc analysis of the WISDOM trial. Lancet Respir Med 2016;4:390-398.

44 Ray A, Oriss TB, Wenzel SE: Emerging molecular phenotypes of asthma. Am J Physiol Lung Cell Mol Physiol 2015;308:L130-L140.

45 Rodrigo GJ, Neffen H, Castro-Rodriguez JA: Efficacy and safety of subcutaneous omalizumab vs placebo as add-on therapy to corticosteroids for children and adults with asthma: a systematic review. Chest 2011;139:28-35.

46 Harris JM, Maciuca R, Bradley MS, et al.: A randomized trial of the efficacy and safety of quilizumab in adults with inadequately controlled allergic asthma. Respir Res 2016;17:29.

47 Ortega HG, Liu MC, Pavord ID, et al.; MENSA Investigators: Mepolizumab treatment in patients with severe eosinophilic asthma. N Engl J Med 2014;371:1198-1207.

48 Bjermer L, Lemiere C, Maspero J, et al.: Reslizumab for inadequately controlled asthma with elevated blood eosinophil levels: a randomized phase 3 study. Chest 2016;150: 789-798.

49 Hanania NA, Korenblat P, Chapman KR, et al.: Efficacy and safety of lebrikizumab in patients with uncontrolled asthma (LAVOLTA I and LAVOLTA II): replicate, phase 3, randomised, double-blind, placebo-controlled trials. Lancet Respir Med 2016;4:781-796.

50 Gonem S, Berair R, Singapuri A, et al.: Fevipiprant, a prostaglandin $\mathrm{D} 2$ receptor 2 antagonist, in patients with persistent eosinophilic asthma: a single-centre, randomised, double-blind, parallel-group, placebo-controlled trial. Lancet Respir Med 2016;4:699-707.

51 Brightling CE, Bleecker ER, Panettieri RA Jr, et al.: Benralizumab for chronic obstructive pulmonary disease and sputum eosinophilia: a randomised, double-blind, placebo-controlled, phase 2a study. Lancet Respir Med 2014;2: 891-901.

52 Cho MH, Castaldi PJ, Hersh CP, et al.; NETT Genetics, ECLIPSE, and COPDGene Investigators: A genome-wide association study of emphysema and airway quantitative imaging phenotypes. Am J Respir Crit Care Med 2015; 192:559-569.

53 Ostridge K, Williams N, Kim V, et al.: Relationship between pulmonary matrix metalloproteinases and quantitative CT markers of small airways disease and emphysema in COPD. Thorax 2016;71:126-132.

54 Busse WW, Holgate S, Kerwin E, et al.: Randomized, double-blind, placebo-controlled study of brodalumab, a human anti-IL-17 receptor monoclonal antibody, in moderate to severe asthma. Am J Respir Crit Care Med 2013;188:1294-1302. 
55 Brusselle GG, Joos G: Is there a role for macrolides in severe asthma? Curr Opin Pulm Med 2014;20:95-102.

56 Castro M, Rubin AS, Laviolette M, et al.: Effectiveness and safety of bronchial thermoplasty in the treatment of severe asthma: a multicenter, randomized, double-blind, sham-controlled clinical trial. Am J Respir Crit Care Med 2010;181:116-124.

57 Medical Research Council Refractory Asthma Stratification Programme in the United Kingdom (RASP-UK): Moving away from the «one size fits all» approach to treatment in severe asthma. www.rasp.org.uk/ (accessed September 1, 2016).
58 Martinez FJ, Foster G, Curtis JL, et al.; NET Research Group: Predictors of mortality in patients with emphysema and severe airflow obstruction. Am J Respir Crit Care Med 2006; 173:1326-1334.

59 Valipour A, Slebos DJ, Herth F, et al.; IMPACT-Study-Team: Endobronchial valve therapy in patients with homogeneous emphysema: results from the IMPACT study. Am J Respir Crit Care Med 2016;194:1073-1082.

60 Busse WW, Trzaskoma B, Omachi TA, et al.: Evaluating Xolair persistency of response after long-term therapy (XPORT). Am J Respir Crit Care Med 2014;189:A6576.
61 Bilano V, Gilmour S, Moffiet T, et al.: Global trends and projections for tobacco use, 19902025: an analysis of smoking indicators from the WHO Comprehensive Information Systems for Tobacco Control. Lancet 2015;385: 966-976.

62 Gordon SB, Bruce NG, Grigg J, et al.: Respiratory risks from household air pollution in low and middle income countries. Lancet Respir Med 2014;2:823-860.

63 Weiss DJ, Casaburi R, Flannery R, et al.: A placebo-controlled, randomized trial of mesenchymal stem cells in COPD. Chest 2013;143: 1590-1598. 